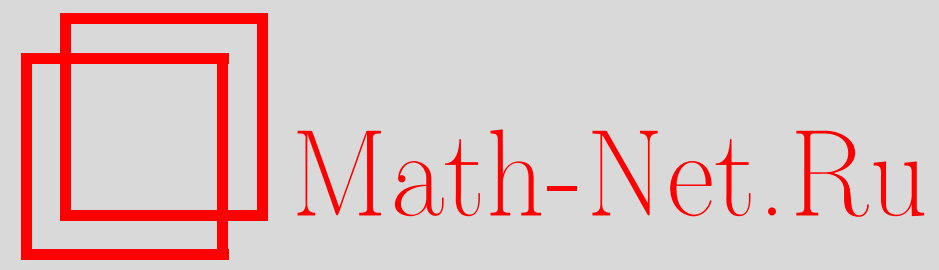

Н. В. Лазакович, С. П. Сташуленок, Аппроксимация стохастических интегралов Ито и Стратоновича элементами прямого произведения алгебр обобщенных случайных процессов, Теория вероятн. и ее примен., 1996, том 41, выпуск 4, 785-809

DOI: https://doi.org/10.4213/tvp3202

Использование Общероссийского математического портала Math-Net.Ru подразумевает, что вы прочитали и согласны с пользовательским соглашением http://www . mathnet.ru/rus/agreement

Параметры загрузки:

IP : 52.6 .47 .48

26 апреля 2023 г., 13:45:34

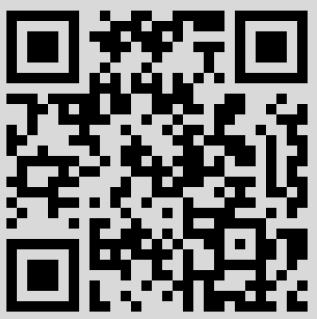


(C) 1996 г. ЛАЗАКОВИЧ Н. В.* , СТАШУЛЕНОК С. П.*

\title{
АППРОКСИМАЦИЯ СТОХАСТИЧЕСКИХ ИНТЕГРАЛОВ ИТО И СТРАТОНОВИЧА ЭЛЕМЕНТАМИ ПРЯМОГО ПРОИЗВЕДЕНИЯ АЛГЕБР ОБОБЩЕННЫХ СЛУЧАЙНЫХ ПРОЦЕССОВ ${ }^{1)}$
}

\begin{abstract}
В статье исследуется задача аппроксимации многомерных стохастических интегралов Ито и Стратоновича конечными суммами элементов из прямого произведения алгебр обобщенных случайных процессов.
\end{abstract}

Ключевые слова и фразы: стандартный процесс броуновского движения, стохастический интеграл Ито, стохастический интеграл Стратоновича, обобщенные случайные процессы, прямое произведение алгебр обобщенных случайных процессов, обобщенный дифференциал Ито, обобщенный дифференциал Стратоновича.

В современной теории дифференциальных уравнений с частными производными основным инструментом служит теория распределений Л. ІІварца, окончательное оформление которой произошло в работе [1]. Однако следует отметить, что с момента своего возникновения теория распределений имела существенный недостаток: она неприменима для решения нелинейных задач. Тем не менее, уже в первых работах П. Дирака, в которых были введены распределения, появиласьь потребность в определении произведения распределений [2]. Многие авторы пытались разрешить эту проблему, используя свои подходы для определения обобщенных функщий. Отметим, в частности, результаты Я. Микусинского $[3],[4]$. Впервые положительное решение было найдено Я. Б. Ливчаком, в работе [5] которого введена алгебра обобщенных функций. Спустя 15 лет она была вновь открыта Ж. Ф. Коломбо [6]. Им и его последователями [7], [8] были исследованы некоторые алгебры объектов, названных ими «новыми обобшенными функциями». Наиболее обширная из такого сорта алгебр была предложена и изучена Ю. В. Егоровым [9]. В статье [10] был предложен общий подход к построению алгебр обобщенных функций.

*Белорусский государственный университет, механико-математический факультет, пр. Ф. Скорины, 4, 220080 Минск, Республика Беларусь.

1) Работа выполнена при поддержке Фонда фундаментальных исследований Республики Беларусь и Международной соросовской программы образования в области точных наук. 
Параллельно с развитием теории обобщенных функций шло развитие теории обобщенных случайных прощессов. Так, на основе теории распределений Л. Шварща И. М. Гельфандом и Н. Я. Виленкиным в [11], [12] создана теория обобшенных случайных процессов. К. Урбаником в статье [13] предложена своя трактовка обобщенных случайных прогессов, опираюшаяся на секвенциальный подход Я. Микусинского. В работе [14] на основе алгебры обобщенных функций Ю. В. Егорова введена алгебра обобщенных случайных процессов. А в [15] показано, что решения стохастических дифференциальных уравнений как Ито, так и Стратоновича могут быть аппроксимированы решением соответствующего уравнения в дифференциалах из данной алгебры.

В настоящей работе исследуются вопросы аппроксимащии многомерных стохастических интегралов Ито и Стратоновича конечными суммами элементов из прямого произведения алгебр обобщенных случайных процессов.

Введение. Напомним некоторые понятия из работы [14], с которыми нам придется работать в дальнейшем.

Пусть $(\Omega, \mathcal{A}, \mathbf{P})$ - полное вероятностное пространство, $T=[0, a] \subset$ $\mathbf{R},\left\{\mathcal{F}_{t}\right\}_{t \in T}-$ стандартный поток $\sigma$-алгебр, $\mathcal{F}_{a} \subset \mathcal{A}$.

Рассмотрим множество функций

$$
f_{n}(t, \omega): \mathbf{N} \times T \times \Omega \longrightarrow \mathbf{R}
$$

таких, что 1) $f_{n}(t, \omega)$ - случайная величина на $(\Omega, \mathcal{A}, \mathbf{P})$ для любых $t \in T$ и $n \in \mathbf{N} ; 2) f_{n}(t, \omega) \in C^{\infty}(T)$ для любых $n \in \mathbf{N}$ и почти всех $\omega \in \Omega$.

Элементы $F=\left(f_{n}(t, \omega)\right)$ и $G=\left(g_{n}(t, \omega)\right)$ данного множества назовем эквивалентными, если сушествует такое $n_{0}$, что для любого $t \in T$, почти всех $\omega \in \Omega$ и любого $n>n_{0}: f_{n}(t, \omega)=g_{n}(t, \omega)$.

Множество классов эквивалентных последовательностей такого вида обозначим $\mathcal{G}(T, \Omega)$, оно является алгеброй с покоординатными операпиями сложения и умножения. Далее, пусть

$$
\widetilde{T}=\left\{\tilde{t}=\left[\left(t_{n}\right)\right] \in \widetilde{\mathbf{R}}: \forall\left(t_{n}\right) \in \tilde{t} \quad 0 \leqslant t_{n} \leqslant a, n=1,2, \ldots\right\},
$$

где $\widetilde{\mathbf{R}}$ - расширенная прямая из [9]. Через $\mathcal{G}(\widetilde{T}, \Omega)$ обозначим алтебру обобценных случайных процессов $\widetilde{F}(\tilde{t}, \omega)$ вида

$$
\tilde{F}(\tilde{t}, \omega)=\left[\left(f_{n}\left(t_{n}, \omega\right)\right)\right]
$$

где $\tilde{t}=\left[\left(t_{n}\right)\right] \in \widetilde{T}$, а $\left[\left(f_{n}(t, \omega)\right)\right] \in \mathcal{G}(T, \Omega)$.

Введем на $\widetilde{\mathbf{R}}$ множество

$$
H=\left\{\widetilde{h}=\left[\left(h_{n}\right)\right] \in \widetilde{\mathbf{R}}_{+}|\widetilde{0}| \lim _{n \rightarrow \infty} h_{n}=0\right\}
$$

и для любого $\widetilde{F}=\left[\left(f_{n}\right)\right] \in \mathcal{G}(\widetilde{T}, \Omega)$ положим по определению

$$
d_{\widetilde{h}} \widetilde{F}(\tilde{t}, \omega)=\left[\left(f_{n}\left(t+h_{n}, \omega\right)-f_{n}(t, \omega)\right)\right],
$$


где $\tilde{t}=[(t)] \in \tilde{T}, \tilde{h} \in H, \tilde{t}+\tilde{h} \in \widetilde{T}$.

Выделим в $H$ подмножества

$$
\begin{aligned}
& I=\left\{\widetilde{h} \in H \mid \frac{1}{n}=o\left(h_{n}\right) \forall\left(h_{n}\right) \in \widetilde{h}\right\}, \\
& S=\left\{\tilde{h} \in H \mid h_{n}=o\left(\frac{1}{n}\right) \forall\left(h_{n}\right) \in \widetilde{h}\right\} .
\end{aligned}
$$

Напомним, что $\boldsymbol{d}_{\widetilde{h}} \widetilde{\boldsymbol{F}}, \widetilde{h} \in S$, называется обобщенным дифференциалом Стратоновича, а $d_{\widetilde{h}} \widetilde{F}, \widetilde{h} \in I,-$ обобщенным цифференциалом Ито.

1. Вспомогательные утверждения. Пусть $B(t, \omega), \quad t \in T$, $\omega \in \Omega$, - одномерный стандартный процесс $\mathcal{F}_{t}$-броуновского движения $[16$, c. $48-49] ; 0=t_{0}<t_{1}<\cdots<t_{m}=a-$ разбиение отрезка $T$, $\lambda_{m}=\max _{1 \leqslant k \leqslant m}\left(t_{k}-t_{k-1}\right)$. Тогда для любого $t \in T$ существует такое $p=p(t) \in \mathbf{N}$, что $t_{p} \leqslant t<t_{p+1}$.

Через $\rho(s), s \in \mathbf{R}$, обозначим неотрицательную функцию класса $C^{\infty}(\mathbf{R})$, носитель которой содержится в $[0,1]$ и $\int_{0}^{1} \rho(s) d s=1$, а $\rho_{n}(s)=$ $n \rho(n s), n \in \mathbf{N}$,

$$
\begin{aligned}
& B_{n}(t, \omega)=\left(B * \rho_{n}\right)(t, \omega)=\int_{0}^{1 / n} B(t+s, \omega) \rho_{n}(s) d s \\
& S_{n}^{p}(t, \omega)=\sum_{k=1}^{p}\left[B_{n}\left(t_{k}, \omega\right)-B_{n}\left(t_{k-1}, \omega\right)\right]^{2}
\end{aligned}
$$

Лемма 1. Справедливы следующие оченки:

a) $\mathbf{E}\left[B_{n}\left(t_{k}, \omega\right)-B_{n}\left(t_{k-1}, \omega\right)\right]^{2} \leqslant C\left|t_{k}-t_{k-1}\right|$,

б) $\mathbf{E}\left[B_{n}(t, \omega)-B(t, \omega)\right]^{2} \leqslant \frac{C}{n}$

в) $\mathbf{E}\left[B_{n}\left(t_{k}, \omega\right)-B_{n}\left(t_{k-1}, \omega\right)\right]^{4} \leqslant C\left(t_{k}-t_{k-1}\right)^{2}$,

г) $\mathbf{E}\left[B_{n}(t, \omega)-B(t, \omega)\right]^{4} \leqslant \frac{C}{n^{2}}$.

Д о к а з а т е л ь с в о использует неравенство Гёльдера и теорему Фубини $[17$, с. 26,30$]$.

Без существенного ограничения общности в дальнейшем предполагаем, что $t_{k}-t_{k-1}=h_{n}, k=1, \ldots, m$.

Лемма 2. Для достаточно больших $n u h_{n}<1 / n$ справедливь следующие оченки:

a) $\mathbf{E}\left[B_{n}\left(t_{k}, \omega\right)-B_{n}\left(t_{k-1}, \omega\right)\right]^{2} \leqslant C n h_{n}^{2}$,

б) $\mathrm{E}\left[B_{n}\left(t_{k}, \omega\right)-B_{n}\left(t_{k-1}, \omega\right)\right]^{4} \leqslant C n^{2} h_{n}^{4}$,

в) $\mathbf{E}\left[B_{n}\left(t_{k}, \omega\right)-B_{n}\left(t_{k-1}, \omega\right)\right]^{8} \leqslant C n^{4} h_{n}^{8}$. 
Д о ка за т е л с т в о проведем для случая б). Используя интегрирование по частям и замену переменных, разность $B_{n}\left(t_{k}, \omega\right)$ $B_{n}\left(t_{k-1}, \omega\right)$ перепишем в виде

$$
\begin{aligned}
B_{n}( & \left.t_{k}, \omega\right)-B_{n}\left(t_{k-1}, \omega\right)=\int_{0}^{1 / n} B\left(t_{k}+s, \omega\right) \rho_{n}(s) d s \\
& -\int_{0}^{1 / n} B\left(t_{k-1}+s, \omega\right) \rho_{n}(s) d s \\
= & \int_{0}^{1 / n}\left[\int_{t_{k}}^{s+t_{k}} B(u, \omega) d u-\int_{t_{k-1}}^{s+t_{k-1}} B(u, \omega) d u\right] \rho_{n}^{\prime}(s) d s \\
= & \int_{0}^{h_{n}}\left[\int_{0}^{s}\left[B\left(t_{k}+u, \omega\right)-B\left(t_{k-1}+u, \omega\right)\right] d u\right] \rho_{n}^{\prime}(s) d s \\
& +\int_{h_{n}}^{1 / n}\left[\int_{t_{k-1}}^{t_{k}}[B(u+s, \omega)-B(u, \omega)] d u\right] \rho_{n}^{\prime}(s) d s=I_{1}^{n}+I_{2}^{n}
\end{aligned}
$$

Для $\mathbf{E}\left[I_{2}^{n}\right]^{4}$ используем неравенство Гёльдера и теорему Фубини. В результате получим

$$
\begin{aligned}
\mathbf{E}\left[I_{2}^{n}\right]^{4} \leqslant & \mathbf{E}\left(\int_{h_{n}}^{1 / n} \int_{t_{k-1}}^{t_{k}}[B(u+s, \omega)-B(u, \omega)]^{4} d u\left(\int_{t_{k-1}}^{t_{k}} d u\right)^{3} d s\right) \\
& \times \int_{h_{n}}^{1 / n} d s\left(\int_{h_{n}}^{1 / n}\left[\rho_{n}^{\prime}(s)\right]^{2} d s\right)^{2} \leqslant C n^{2} h_{n}^{4} .
\end{aligned}
$$

Рассуждая аналогично, для $\mathbf{E}\left[I_{1}^{n}\right]^{4}$ получим

$$
\begin{aligned}
\mathrm{E}\left[I_{1}^{n}\right]^{4} \leqslant & \mathrm{E} \int_{0}^{h_{n}} \int_{0}^{s}\left[B\left(t_{k}+u, \omega\right)-B\left(t_{k+1}+u, \omega\right)\right]^{4} d u\left(\int_{0}^{s} d u\right)^{3} d s \\
& \times \int_{0}^{h_{n}} d s\left[\int_{0}^{h_{n}}\left[\rho_{n}^{\prime}(s)\right]^{2} d s\right]^{2} \leqslant C n^{8} h_{n}^{10} .
\end{aligned}
$$

Используя неравенство

$$
\mathbf{E}\left[I_{1}^{n}+I_{2}^{n}\right]^{4} \leqslant C \mathbf{E}\left[I_{1}^{n}\right]^{4}+C \mathbf{E}\left[I_{2}^{n}\right]^{4}
$$

и тот факт, что $h_{n}<1 / n$, получаем утверждение б) леммы 2 .

Аналогично доказываются пункты а) и в).

Лемма 3. Eсли $n \rightarrow \infty, h_{n} \rightarrow 0$ mak, ито $1 / n=o\left(h_{n}\right)$, mo

$$
\sup _{t \in T} \mathrm{E}\left[S_{n}^{p}(t, \omega)-t\right]^{2} \longrightarrow 0
$$

Д о к а за т е л с т в о. Очевидно следуюшее неравенство: 


$$
\begin{aligned}
& \mathbf{E}\left[\sum_{k=1}^{p}\left[B_{n}\left(t_{k}, \omega\right)-B_{n}\left(t_{k-1}, \omega\right)\right]^{2}-t\right]^{2} \\
& \quad \leqslant 2 \mathbf{E}\left[\sum_{k=1}^{p}\left[B_{n}\left(t_{k}, \omega\right)-B_{n}\left(t_{k-1}, \omega\right)\right]^{2}-\sum_{k=1}^{p}\left[B\left(t_{k}, \omega\right)-B\left(t_{k-1}, \omega\right)\right]^{2}\right]^{2} \\
& \quad+2 \mathbf{E}\left[\sum_{k=1}^{p}\left[B\left(t_{k}, \omega\right)-B\left(t_{k-1}, \omega\right)\right]^{2}-t\right]^{2}=2 I_{1}(t)+2 I_{2}(t) .
\end{aligned}
$$

Известно [18, с. 19], что если $h_{n} \rightarrow 0$, то

$$
\sup _{t \in T} I_{2}(t) \longrightarrow 0 \text {. }
$$

Справедлива следующая оценка для $I_{1}(t)$ :

$$
\begin{aligned}
I_{1}(t) \leqslant & 4 \mathbf{E}\left[\sum_{k=1}^{p}\left(B_{n}\left(t_{k}, \omega\right)-B\left(t_{k}, \omega\right)\right)\left(B_{n}\left(t_{k}, \omega\right)-B_{n}\left(t_{k-1}, \omega\right)\right)\right]^{2} \\
& +4 \mathbf{E}\left[\sum_{k=1}^{p}\left(B_{n}\left(t_{k-1}, \omega\right)-B\left(t_{k-1}, \omega\right)\right)\left(B_{n}\left(t_{k}, \omega\right)-B_{n}\left(t_{k-1}, \omega\right)\right)\right]^{2} \\
& +4 \mathbf{E}\left[\sum_{k=1}^{p}\left(B_{n}\left(t_{k}, \omega\right)-B\left(t_{k}, \omega\right)\right)\left(B\left(t_{k}, \omega\right)-B\left(t_{k-1}, \omega\right)\right)\right]^{2} \\
& +4 \mathbf{E}\left[\sum_{k=1}^{p}\left(B_{n}\left(t_{k-1}, \omega\right)-B\left(t_{k-1}, \omega\right)\right)\left(B\left(t_{k}, \omega\right)-B\left(t_{k-1}, \omega\right)\right)\right]^{2} \\
= & 4 J_{1}(t)+4 J_{2}(t)+4 J_{3}(t)+4 J_{4}(t) .
\end{aligned}
$$

Используя неравенство Гёльдера, мартингальное неравенство [16, c. 41] и пункт в) леммы 1 , для $J_{1}(t)$ можно получить следующую огенку:

$$
J_{1}(t) \leqslant \frac{C p^{2} h_{n}^{2}}{n} \leqslant \frac{C}{n} .
$$

Аналогично доказывается, что

$$
J_{2}(t) \leqslant \frac{C}{n}
$$

Рассмотрим $J_{3}(t)$. Используя лемму 1 , получим

$$
\begin{aligned}
J_{3}(t) \leqslant & p \sum_{k=1}^{p}\left(\mathrm{E}\left(B_{n}\left(t_{k}, \omega\right)-B\left(t_{k}, \omega\right)\right)^{4}\right)^{1 / 2} \\
& \times\left(\mathrm{E}\left(B\left(t_{k}, \omega\right)-B\left(t_{k-1}, \omega\right)\right)^{4}\right)^{1 / 2} \leqslant \frac{C p^{2} h_{n}}{n} \leqslant \frac{C}{n h_{n}}
\end{aligned}
$$


Точно так же доказывается, что

$$
J_{4}(t) \leqslant \frac{C}{n h_{n}}
$$

Таким образом, из соотношений (1.2)-(1.8) вытекает доказательство леммы.

Лемма 4. $E c л и h_{n}=o(1 / n)$ nрu $n \rightarrow \infty$, mo

$$
\sup _{t \in T} \mathbf{E}\left[S_{n}^{p}(t, \omega)\right]^{2} \longrightarrow 0 \text {. }
$$

Д оказа т е л с т в о. Используя пункт б) леммы 2, получаем

$$
\begin{aligned}
\mathbf{E}\left[S_{n}^{p}(t, \omega)\right]^{2} & \leqslant p \sum_{k=1}^{p} \mathbf{E}\left[B_{n}\left(t_{k}, \omega\right)-B_{n}\left(t_{k-1}, \omega\right)\right]^{4} \\
& \leqslant p \sum_{k=1}^{p} C n^{2} h_{n}^{4} \leqslant C n^{2} h_{n}^{2} \longrightarrow 0, \quad n \rightarrow \infty .
\end{aligned}
$$

Лемма 5 [19, с. 73]. Предположим, ито $C>1$ постоянна. Тогда длg почти всех $\omega$ существует такое $\delta=\delta(\omega)>0$, ито $n p u\left|t-t^{\prime}\right| \leqslant \delta$

$$
\left|B(t, \omega)-B\left(t^{\prime}, \omega\right)\right| \leqslant C\left[2\left|t-t^{\prime}\right| \log \left|t-t^{\prime}\right|^{-1}\right]^{1 / 2}
$$

Используя методику доказательства лемм 3 и 4, а также лемму 5, можно убедиться в справедливости следующих утверждений.

Лемма 6. Если для любого сколь угодно малого $\alpha>0$ при $n \rightarrow \infty$, $m \rightarrow \infty, \lambda_{m} \rightarrow 0: m / n^{1-\alpha} \rightarrow 0$, то равномерно по $t \in T$ для почти всех $\omega \in \Omega$

$$
S_{n}^{p}(t, \omega)-t \longrightarrow 0 \text {. }
$$

Лемма 7. Если для любого сколь угодно малого $\alpha>0$ при $n \rightarrow \infty$,

$$
\lambda_{m} n^{1+\alpha} \longrightarrow 0,
$$

то равномерно по $t \in T$ для почти всех $\omega \in \Omega$

$$
S_{n}^{p}(t, \omega) \longrightarrow 0 \text {. }
$$

2. Аппроксимация стохастического интеграла Ито в прямом произведении алгебр обобщенных случайных процессов. Введем $r$-мерный стандартный прощесс $\mathcal{F}_{t}$-броуновского движения $\bar{B}(t, \omega)=\left(B^{1}(t, \omega), \ldots, B^{r}(t, \omega)\right), t \in T, \omega \in \Omega,[16$, с. 48-49] и через

$$
\text { (I) } \int_{0}^{t} u(\bar{B}(s, \omega)) d B^{i}(s, \omega), \quad t \in T, \quad \omega \in \Omega, \quad i=1, \ldots, r
$$

обозначим стохастический интеграл Ито [16, с. 53];

$C_{B}^{l}\left(\mathbf{R}^{r}\right)$ - множество действительных $l$ раз непрерывно дифференцируемых на $\mathbf{R}^{r}$ функций, ограниченных вместе со своими частными производными до порядка $l$ включительно; 
$\mathcal{G}(T, \Omega)^{r}, \mathcal{G}(\widetilde{T}, \Omega)^{r}$ - прямое произведение алгебр $\mathcal{G}(T, \Omega)$ и $\mathcal{G}(\widetilde{T}, \Omega)$ соответственно.

В данном пункте исследуется задача аппроксимации стохастического интеграла (2.1) конечными суммами элементов из прямого произведения алгебр обобщенных случайных процессов. Известно [20], что даже для «хороших》 случайных функций $u(B)$ соответствующие «приближенные» интегралы не сходятся к стохастическому интегралу Ито. В работе [21] указанные выше трудности для некоторых аппроксимаций броуновского движения удалось преодолеть лишь с введением запаздывания. В рассматриваемом прямом произведении алгебр подобной ситуапии не возникает.

Теорема 1. Для пюбой функции $u \in C_{B}^{1}\left(\mathbf{R}^{r}\right)$ u r-мерного стандартного случайного прочесса броуновского движения найдутся такие элементы $\widetilde{u} \in \mathcal{G}\left(\mathbf{R}^{r}\right)$ и $\widetilde{B} \in \mathcal{G}(\tilde{T}, \Omega)^{r}$, что для любых представителей $\left(u_{n}\right) \in \widetilde{u} u\left(\bar{B}_{n}(s, \omega)\right) \in \widetilde{B}$ справедливо неравенство

$$
\begin{aligned}
\sup _{t \in T} \mathbf{E}\left\{\sum_{k=1}^{p} \sum_{i=1}^{r} u_{n}\left(B_{n}\left(t_{k-1}, \omega\right)\right)\left[B_{n}^{i}\left(t_{k}, \omega\right)-B_{n}^{i}\left(t_{k-1}, \omega\right)\right]\right. \\
\left.-\sum_{i=1}^{r}(\mathrm{I}) \int_{0}^{t} u(\bar{B}(s, \omega)) d B^{i}(s, \omega)\right\}^{2} \leqslant \frac{C m}{n}+C \lambda_{m},
\end{aligned}
$$

если $1 / n<\min _{1 \leqslant k \leqslant m}\left(t_{k}-t_{k-1}\right)$, где $\bar{B}_{n}=\left(B_{n}^{1}, \ldots, B_{n}^{r}\right)$.

Д ок азате ль с т в о. Для простоты исследуем случай $r=2$. Достаточно будет рассмотреть первое слагаемое. В качестве представителя функции $\widetilde{u}$ выберем

$$
u_{n}\left(t_{1}, t_{2}\right)=\int_{0}^{1 / n} \int_{0}^{1 / n} u\left(t_{1}+s_{1}, t_{2}+s_{2}\right) \bar{\rho}_{n}\left(s_{1}, s_{2}\right) d s_{1} d s_{2}
$$

где

$$
\bar{\rho}_{n}\left(s_{1}, s_{2}\right)=n^{2} \bar{\rho}\left(n s_{1}, n s_{2}\right),
$$

а $\bar{\rho}\left(s_{1}, s_{2}\right)$ - неотрицательная бесконечно дифференцируемая функция, носитель которой содержится в квацрате $[0,1]^{2}$, и $\int_{[0,1]^{2}} \bar{\rho} d s_{1} d s_{2}=1$; а в качестве представителя $\widetilde{B}^{i}$ выберем

$$
B_{n}^{i}(t, \omega)=\left(B^{i} * \rho_{n}\right)(t, \omega), \quad i=1, \ldots, r .
$$

Используя неравенство треугольника для нормы в $L^{2}(\Omega, \mathcal{A}, \mathbf{P})$, несложно видеть, что для произвольного $t \in T$

$$
\begin{gathered}
\left\{\mathbf { E } \left\{\sum_{k=1}^{p} u_{n}\left(B_{n}^{1}\left(t_{k-1}, \omega\right), B_{n}^{2}\left(t_{k-1}, \omega\right)\right)\left[B_{n}^{1}\left(t_{k}, \omega\right)-B_{n}^{1}\left(t_{k-1}, \omega\right)\right]\right.\right. \\
\left.\left.-(\mathrm{I}) \int_{0}^{t} u\left(B^{1}(s, \omega), B^{2}(s, \omega)\right) d B^{1}(s, \omega)\right\}^{2}\right\}^{1 / 2}
\end{gathered}
$$




$$
\begin{aligned}
& \leqslant\left\{\mathrm { E } \left\{\sum_{k=1}^{p} u_{n}\left(B_{n}^{1}\left(t_{k-1}, \omega\right), B_{n}^{2}\left(t_{k-1}, \omega\right)\right)\left[B_{n}^{1}\left(t_{k}, \omega\right)-B_{n}^{1}\left(t_{k-1}, \omega\right)\right]\right.\right. \\
& -\sum_{k=1}^{p} u_{n}\left(B_{n}^{1}\left(t_{k-1}-\frac{1}{n}, \omega\right), B_{n}^{2}\left(t_{k-1}-\frac{1}{n}, \omega\right)\right) \\
& \left.\left.\times\left[B_{n}^{1}\left(t_{k}, \omega\right)-B_{n}^{1}\left(t_{k-1}, \omega\right)\right]\right\}^{2}\right\}^{1 / 2} \\
& +\left\{\mathbf { E } \left\{\sum_{k=1}^{p} u_{n}\left(B_{n}^{1}\left(t_{k-1}-\frac{1}{n}, \omega\right), B_{n}^{2}\left(t_{k-1}-\frac{1}{n}, \omega\right)\right)\right.\right. \\
& \times\left[B_{n}^{1}\left(t_{k}, \omega\right)-B_{n}^{1}\left(t_{k-1}, \omega\right)\right] \\
& \left.\left.-(\mathrm{I}) \int_{0}^{t} u\left(B^{1}(s, \omega), B^{2}(s, \omega)\right) d B^{1}(s, \omega)\right\}^{2}\right\}^{1 / 2} \\
& =I_{1}^{n, p}(t)+I_{2}^{n, p}(t) .
\end{aligned}
$$

Используя это же неравенство, для $I_{2}^{n, p}(t)$ получаем:

$$
\begin{aligned}
& I_{2}^{n, p}(t) \leqslant\left\{\mathbf { E } \left\{\sum_{k=1}^{p} u_{n}\left(B_{n}^{1}\left(t_{k-1}-\frac{1}{n}, \omega\right), B_{n}^{2}\left(t_{k-1}-\frac{1}{n}, \omega\right)\right)\right.\right. \\
& \times\left[B_{n}^{1}\left(t_{k}, \omega\right)-B_{n}^{1}\left(t_{k-1}, \omega\right)\right] \\
& -\sum_{k=1}^{p} u_{n}\left(B^{1}\left(t_{k-1}-\frac{1}{n}, \omega\right), B^{2}\left(t_{k-1}-\frac{1}{n}, \omega\right)\right) \\
& \left.\left.\times\left[B_{n}^{1}\left(t_{k}, \omega\right)-B_{n}^{1}\left(t_{k-1}, \omega\right)\right]\right\}^{2}\right\}^{1 / 2} \\
& +\left\{\mathrm { E } \left\{\sum_{k=1}^{p} u_{n}\left(B^{1}\left(t_{k-1}-\frac{1}{n}, \omega\right), B^{2}\left(t_{k-1}-\frac{1}{n}, \omega\right)\right)\right.\right. \\
& \times\left[B_{n}^{1}\left(t_{k}, \omega\right)-B_{n}^{1}\left(t_{k-1}, \omega\right)\right] \\
& -\sum_{k=1}^{p} u\left(B^{1}\left(t_{k-1}-\frac{1}{n}, \omega\right), B^{2}\left(t_{k-1}-\frac{1}{n}, \omega\right)\right) \\
& \left.\left.\times\left[B_{n}^{1}\left(t_{k}, \omega\right)-B_{n}^{1}\left(t_{k-1}, \omega\right)\right]\right\}^{2}\right\}^{1 / 2} \\
& +\left\{\mathrm { E } \left\{\sum_{k=1}^{p} u\left(B^{1}\left(t_{k-1}-\frac{1}{n}, \omega\right), B^{2}\left(t_{k-1}-\frac{1}{n}, \omega\right)\right)\right.\right. \\
& \times\left[B_{n}^{1}\left(t_{k}, \omega\right)-B_{n}^{1}\left(t_{k-1}, \omega\right)\right]
\end{aligned}
$$




$$
\begin{gathered}
-\sum_{k=1}^{p} u\left(B^{1}\left(t_{k-1}-\frac{1}{n}, \omega\right), B^{2}\left(t_{k-1}-\frac{1}{n}, \omega\right)\right) \\
\left.\left.\times\left[B^{1}\left(t_{k}, \omega\right)-B^{1}\left(t_{k-1}, \omega\right)\right]\right\}^{2}\right\}^{1 / 2} \\
+\left\{\mathbf { E } \left\{\sum_{k=1}^{p} u\left(B^{1}\left(t_{k-1}-\frac{1}{n}, \omega\right), B^{2}\left(t_{k-1}-\frac{1}{n}, \omega\right)\right)\right.\right. \\
\times\left[B^{1}\left(t_{k}, \omega\right)-B^{1}\left(t_{k-1}, \omega\right)\right] \\
-\sum_{k=1}^{p} u\left(B^{1}\left(t_{k-1}, \omega\right), B^{2}\left(t_{k-1}, \omega\right)\right) \\
+\left\{\mathbf { E } \left\{\sum_{k=1}^{p} u\left(B^{1}\left(t_{k-1}, \omega\right), B^{2}\left(t_{k-1}, \omega\right)\right)\right.\right. \\
\times\left[B^{1}\left(t_{k}, \omega\right)-B^{1}\left(t_{k-1}, \omega\right)\right] \\
\left.\left.\left.\left.\quad-(\mathrm{I}) \int_{0}^{t} u\left(B^{1}(s, \omega), B^{2}(s, \omega)\right) d B^{1}(s, \omega)\right\}^{1 / 2}\right\}^{2}\left(t_{k-1}, \omega\right)\right]\right\}^{1 / 2} \\
+I_{22}(t)+I_{23}(t)+I_{24}(t)+I_{25}(t)
\end{gathered}
$$

Для оценки $I_{21}(t)$ будем использовать оценку в) из леммы 1 , неравенство Гёльдера, теорему Лагранжа и ограниченность $\partial u_{n} / \partial x_{i}$ :

$$
\begin{aligned}
& I_{21}^{2}(t) \leqslant p \sum_{k=1}^{p} \mathbf{E}\{ {\left[u_{n}\left(B_{n}^{1}\left(t_{k-1}-\frac{1}{n}, \omega\right), B_{n}^{2}\left(t_{k-1}-\frac{1}{n}, \omega\right)\right)\right.} \\
&\left.-u_{n}\left(B^{1}\left(t_{k-1}-\frac{1}{n}, \omega\right), B^{2}\left(t_{k-1}-\frac{1}{n}, \omega\right)\right)\right] \\
&\left.\times\left[B_{n}^{1}\left(t_{k}, \omega\right)-B_{n}^{1}\left(t_{k-1}, \omega\right)\right]\right\}^{2} \\
& \leqslant C p \sum_{k=1}^{p}\{ \mathbf{E}\left(B_{n}^{1}\left(t_{k-1}-\frac{1}{n}, \omega\right)-B^{1}\left(t_{k-1}-\frac{1}{n}, \omega\right)\right)^{4} \\
&\left.+\mathbf{E}\left(B_{n}^{2}\left(t_{k-1}-\frac{1}{n}, \omega\right)-B^{2}\left(t_{k-1}-\frac{1}{n}, \omega\right)\right)^{4}\right\}^{1 / 2} \\
& \times\left(t_{k}-t_{k-1}\right) \leqslant \frac{C p}{n} .
\end{aligned}
$$

Используя те же фақты, что и при оценивании $I_{21}(t)$, а также пред- 
ставление $u_{n}$, для $I_{22}(t)$ имеем:

$$
\begin{aligned}
& I_{22}^{2}(t) \leqslant p \sum_{k=1}^{p} \mathbf{E}\left\{\left[u_{n}\left(B^{1}\left(t_{k-1}-\frac{1}{n}, \omega\right), B^{2}\left(t_{k-1}-\frac{1}{n}, \omega\right)\right)\right.\right. \\
& \left.-u\left(B^{1}\left(t_{k-1}-\frac{1}{n}, \omega\right), B^{2}\left(t_{k-1}-\frac{1}{n}, \omega\right)\right)\right] \\
& \left.\times\left[B_{n}^{1}\left(t_{k}, \omega\right)-B_{n}^{1}\left(t_{k-1}, \omega\right)\right]\right\}^{2} \\
& \leqslant p \sum_{k=1}^{p}\left\{\mathbf { E } \left[\int _ { 0 } ^ { 1 / n } \int _ { 0 } ^ { 1 / n } \left[u \left(B^{1}\left(t_{k-1}-\frac{1}{n}, \omega\right)+s_{1},\right.\right.\right.\right. \\
& \left.B^{2}\left(t_{k-1}-\frac{1}{n}, \omega\right)+s_{2}\right) \\
& \left.-u\left(B^{1}\left(t_{k-1}-\frac{1}{n}, \omega\right), B^{2}\left(t_{k-1}-\frac{1}{n}, \omega\right)\right)\right] \\
& \left.\left.\times \bar{\rho}_{n}\left(s_{1}, s_{2}\right) d s_{1} d s_{2}\right]^{4} \mathbf{E}\left[B_{n}^{1}\left(t_{k}, \omega\right)-B_{n}^{1}\left(t_{k-1}, \omega\right)\right]^{4}\right\}^{1 / 2} \\
& \leqslant p \sum_{k=1}^{p} C \frac{t_{k}-t_{k-1}}{n^{2}} \leqslant \frac{C p}{n^{2}}
\end{aligned}
$$

Проводя стандартные рассуждения, для $I_{23}(t)$ получаем (здесь используем условие, что $\left.1 / n<\min _{1 \leqslant k \leqslant m}\left(t_{k}-t_{k-1}\right)\right)$ :

$$
\begin{aligned}
I_{23}(t) \leqslant & \left\{\mathbf { E } \left\{\sum_{k=1}^{p} u\left(B^{1}\left(t_{k-1}-\frac{1}{n}, \omega\right), B^{2}\left(t_{k-1}-\frac{1}{n}, \omega\right)\right)\right.\right. \\
& \left.\left.\times\left[B_{n}^{1}\left(t_{k}, \omega\right)-B^{1}\left(t_{k}, \omega\right)\right]\right\}^{2}\right\}^{1 / 2} \\
+ & \left\{\mathbf { E } \left\{\sum_{k=1}^{p} u\left(B^{1}\left(t_{k-1}-\frac{1}{n}, \omega\right), B^{2}\left(t_{k-1}-\frac{1}{n}, \omega\right)\right)\right.\right. \\
& \left.\times\left[B_{n}^{1}\left(t_{k-1}, \omega\right)-B^{1}\left(t_{k-1}, \omega\right)\right]\right\}^{2}=I_{231}(t)+I_{232}(t),
\end{aligned}
$$

где

$$
\begin{gathered}
I_{231}^{2}(t)=\mathrm{E}\left\{\sum_{k=1}^{p} u\left(B^{1}\left(t_{k-1}-\frac{1}{n}, \omega\right), B^{2}\left(t_{k-1}-\frac{1}{n}, \omega\right)\right)\right. \\
\left.\times\left[B_{n}^{1}\left(t_{k}, \omega\right)-B^{1}\left(t_{k}, \omega\right)\right]\right\}^{2}
\end{gathered}
$$




$$
\begin{aligned}
=\sum_{k=1}^{p} \mathbf{E}\{u & \left(B^{1}\left(t_{k-1}-\frac{1}{n}, \omega\right), B^{2}\left(t_{k-1}-\frac{1}{n}, \omega\right)\right) \\
& \left.\times\left[B_{n}^{1}\left(t_{k}, \omega\right)-B^{1}\left(t_{k}, \omega\right)\right]\right\}^{2}
\end{aligned}
$$

Тогда

$$
\begin{aligned}
& I_{231}^{2}(t) \leqslant C \sum_{k=1}^{p} \mathbf{E}\left[B_{n}^{1}\left(t_{k}, \omega\right)-B^{1}\left(t_{k}, \omega\right)\right]^{2} \leqslant \frac{C p}{n} \\
& I_{232}^{2}(t) \leqslant C \sum_{k=1}^{p} \mathbf{E}\left[B_{n}^{1}\left(t_{k-1}, \omega\right)-B^{1}\left(t_{k-1}, \omega\right)\right]^{2} \leqslant \frac{C p}{n} .
\end{aligned}
$$

Как и для $I_{23}(t)$, для $I_{24}(t)$ получаем:

$$
\begin{gathered}
I_{24}^{2}(t) \leqslant \sum_{k=1}^{p} \mathbf{E}\left[u\left(B^{1}\left(t_{k-1}-\frac{1}{n}, \omega\right), B^{2}\left(t_{k-1}-\frac{1}{n}, \omega\right)\right)\right. \\
\left.-u\left(B^{1}\left(t_{k-1}, \omega\right), B^{2}\left(t_{k-1}, \omega\right)\right)\right]^{2} \\
\times \mathbf{E}\left[B^{1}\left(t_{k}, \omega\right)-B^{1}\left(t_{k-1}, \omega\right)\right]^{2} \leqslant \frac{C}{n} .
\end{gathered}
$$

При получении последней оценки использовалась независимость случайных величин $u\left(B^{1}\left(t_{k-1}-1 / n, \omega\right), B^{2}\left(t_{k-1}-1 / n, \omega\right)\right)-u\left(B^{1}\left(t_{k-1}, \omega\right)\right.$, $\left.B^{2}\left(t_{k-1}, \omega\right)\right)$ и $B^{1}\left(t_{k}, \omega\right)-B^{1}\left(t_{k-1}, \omega\right)$, а также теорема Лагранжа, ограниченность $\partial u / \partial x_{i}$ и оценка а) леммы 1 .

Положим по определению $\bar{B}(s, \omega)=B^{i}\left(t_{k-1}, \omega\right)$ для $s \in\left[t_{k-1}, t_{k}\right]$, $k=1, \ldots, m$. Тогда, используя определение стохастического интеграла Ито и изометрию $(4.7)[22$, с. 260$]$, для $I_{25}(t)$ получим:

$$
I_{25}^{2}(t) \leqslant C \lambda_{m}
$$

Из соотношений (2.5)-(2.12) получаем

$$
\left(I_{2}^{n, p}(t)\right)^{2} \leqslant \frac{C p}{n}+\frac{C p}{n^{2}}+\frac{C}{n}+C \lambda_{m}
$$

И, наконеп, для ощенки $I_{1}^{n, p}(t)$ используем неравенство Гёльдера, теорему Лагранжа, ограниченность $\partial u_{n} / \partial x_{i}$, пункты в) и г) леммы 1:

$$
\begin{aligned}
\left(I_{2}^{n, p}(t)\right)^{2} \leqslant p \sum_{k=1}^{p}\{\mathbf{E} & {\left[u_{n}\left(B_{n}^{1}\left(t_{k-1}, \omega\right), B_{n}^{2}\left(t_{k-1}, \omega\right)\right)\right.} \\
& \left.-u_{n}\left(B_{n}^{1}\left(t_{k-1}-\frac{1}{n}, \omega\right), B_{n}^{2}\left(t_{k-1}-\frac{1}{n}, \omega\right)\right)\right]^{4} \\
& \left.\times \mathbf{E}\left[B_{n}^{1}\left(t_{k}, \omega\right)-B_{n}^{1}\left(t_{k-1}, \omega\right)\right]^{4}\right\}^{1 / 2}
\end{aligned}
$$




$$
\leqslant \frac{C p}{n} \sum_{k=1}^{p}\left(t_{k}-t_{k-1}\right) \leqslant \frac{C p}{n}
$$

Таким образом, для произвольного $t \in T$ получаем

$$
\begin{aligned}
& \mathrm{E}\left\{\sum_{k=1}^{p} u_{n}\left(B_{n}^{1}\left(t_{k-1}, \omega\right), B_{n}^{2}\left(t_{k-1}, \omega\right)\right)\left[B_{n}^{1}\left(t_{k}, \omega\right)-B_{n}^{1}\left(t_{k-1}, \omega\right)\right]\right. \\
& \left.\quad-(\mathrm{I}) \int_{0}^{t} u\left(B^{1}(s, \omega), B^{2}(s, \omega)\right) d B^{1}(s, \omega)\right\}^{2} \\
& \quad \leqslant \frac{C p}{n}+\frac{C p}{n^{2}}+\frac{C}{n}+C \lambda_{m}
\end{aligned}
$$

Отсюда следует утверждение теоремы.

Следствие. Пусть выполнены условия теоремы 1. Тогда, если $n \rightarrow \infty, m \rightarrow \infty, \lambda_{m} \rightarrow 0 \max$, чmo $m / n \rightarrow 0$, mo

$$
\begin{gathered}
\sup _{t \in T} \mathbf{E}\left\{\sum_{i=1}^{r} \sum_{k=1}^{p} u_{n}\left(\bar{B}_{n}\left(t_{k-1}, \omega\right)\right)\left[B_{n}^{i}\left(t_{k}, \omega\right)-B_{n}^{i}\left(t_{k-1}, \omega\right)\right]\right. \\
\left.-\sum_{i=1}^{r}(\mathrm{I}) \int_{0}^{t} u(\bar{B}(s, \omega)) d B^{i}(s, \omega)\right\}^{2} \longrightarrow 0
\end{gathered}
$$

Теорема 2. Пусть функция $u \in C\left(\mathbf{R}^{r}\right)$ u $\bar{B}(t, \omega)$ есть $r$-мерный стандартный случайный прочесс $\mathcal{F}_{t}$-броуновского движения. Тогда найдутся такие элементы $\widetilde{u} \in \mathcal{G}\left(\mathbf{R}^{r}\right)$ u $\widetilde{B} \in \mathcal{G}(\widetilde{T}, \Omega)^{r}$, ито если для любого сколь угодно малого $\alpha>0$ при $n \rightarrow \infty, m \rightarrow \infty, \lambda_{m} \rightarrow 0$

$$
\frac{m}{n^{1-\alpha}} \longrightarrow 0
$$

mo

$$
\begin{gathered}
\sup _{t \in T} \mid \sum_{i=1}^{r} \sum_{k=1}^{p} u_{n}\left(\bar{B}_{n}\left(t_{k-1}, \omega\right)\right)\left[B_{n}^{i}\left(t_{k}, \omega\right)-B_{n}^{i}\left(t_{k-1}, \omega\right)\right] \\
-\sum_{i=1}^{r}(\mathrm{I}) \int_{0}^{t} u(\bar{B}(s, \omega)) d B^{i}(s, \omega) \mid \longrightarrow 0
\end{gathered}
$$

для почти всех $\omega \in \Omega$. Здесь $\left(u_{n}\right) u\left(\bar{B}_{n}(s, \omega)\right)$ - произвольные представители $\widetilde{u}$ и $\widetilde{B}$ соответственно, $C^{l}\left(\mathbf{R}^{r}\right)$ - множество вешественных $l$ раз непрерывно дифференцируемых на $\mathbf{R}^{r}$ функчий.

Д ок а зате ль с т в т е о р м ы 2 проводится по методике, предложенной в доказательстве теоремы 1 с использованием леммы 5. 
3. Аппроксимация стохастического интеграла Стратоновича в прямом произведении алгебр обобщенных случайных процессов. Пусть

$$
\text { (S) } \int_{0}^{t} u(\bar{B}(s, \omega)) d B^{i}(s, \omega), \quad t \in T, \quad \omega \in \Omega, \quad i=1, \ldots, r
$$

- стохастический интеграл Стратоновича [16, с. 109]. В этом пункте изучается аппроксимация стохастического интеграла (3.1) конечными суммами элементов из прямого произведения алгебр обобщеныых случайных процессов.

Теорема 3. Для любой функчии $u \in C_{B}^{2}\left(\mathbf{R}^{r}\right)$ u $r$-мерного стандартного случайного процесса $\mathcal{F}_{t}$-броуновского движения $\bar{B}(t, \omega)$ найдутся такие элементы $\widetilde{u} \in \mathcal{G}\left(\mathbf{R}^{r}\right)$ u $\widetilde{B} \in \mathcal{G}(\widetilde{T}, \Omega)^{r}$, что для любых представителей $\left(u_{n}\right) \in \widetilde{u} u\left(\bar{B}_{n}\right) \in \widetilde{B}$ справедливо неравенство

$$
\begin{array}{rl}
\sup _{t \in T} & \mathrm{E}\left\{\sum_{j=1}^{r} \sum_{k=1}^{p} u_{n}\left(\bar{B}_{n}\left(t_{k-1}, \omega\right)\right)\left[B_{n}^{j}\left(t_{k}, \omega\right)-B_{n}^{j}\left(t_{k-1}, \omega\right)\right]\right. \\
& \left.-\sum_{j=1}^{r}(\mathrm{~S}) \int_{0}^{t} u(\bar{B}(s, \omega)) d B^{j}(s, \omega)\right\}^{2} \leqslant \frac{C}{n^{1 / 3}}+C n^{2} h_{n}^{2}+C h_{n} .
\end{array}
$$

Доказательство. Введем обозначения. Выберем $N=$ $N\left(h_{n}\right):(0,1) \longrightarrow \mathbf{Z}^{+}$такое, что $h_{n} N\left(h_{n}\right)=\tilde{\delta} \longrightarrow 0, N\left(h_{n}\right) \rightarrow \infty, 1 / n=o(\tilde{\delta})$ при $h_{n} \rightarrow 0$. Без сушественного ограничения общности предположим, что $t=p h_{n}=N M h_{n}=M \tilde{\delta}$. Всюду в дальнейшем в доказательстве теоремы будем предполагать, что $h_{n}=o(1 / n)$.

Представителей $\widetilde{u}$ и $\widetilde{B}$ определим посредством равенств (2.2) и (2.4) соответственно.

Используя связь между интегралами Ито и Стратоновича, получим:

$$
\begin{aligned}
\sum_{k=1}^{p} u_{n}\left(\bar{B}_{n}\left(t_{k-1}, \omega\right)\right)\left[B_{n}^{j}\left(t_{k}, \omega\right)-B_{n}^{j}\left(t_{k-1}, \omega\right)\right] \\
-(\mathrm{S}) \int_{0}^{t} u(\bar{B}(s, \omega)) d B^{j}(s, \omega) \\
=\left\{\sum_{l=1}^{M} u_{n}\left(\bar{B}_{n}((l-1) \tilde{\delta}, \omega)\right)\left[B_{n}^{j}(l \tilde{\delta}, \omega)-B_{n}^{j}((l-1) \tilde{\delta}, \omega)\right]\right. \\
\left.-(\mathrm{I}) \int_{0}^{t} u(\bar{B}(s, \omega)) d B^{j}(s, \omega)\right\} \\
+\left\{\sum _ { l = 1 } ^ { M } \sum _ { k = 1 } ^ { N } \left[u_{n}\left(\bar{B}_{n}\left((l-1) \tilde{\delta}+(k-1) h_{n}, \omega\right)\right)\right.\right.
\end{aligned}
$$




$$
\begin{gathered}
\left.-u_{n}\left(\bar{B}_{n}((l-1) \tilde{\delta}, \omega)\right)\right] \\
\times\left[B_{n}^{j}\left((l-1) \tilde{\delta}+k h_{n}, \omega\right)-B_{n}^{j}\left((l-1) \tilde{\delta}+(k-1) h_{n}, \omega\right)\right] \\
\left.-\frac{1}{2} \int_{0}^{t} u_{j}(\bar{B}(s, \omega)) d s\right\}=I_{1}+I_{2},
\end{gathered}
$$

где $u_{j}=\partial u / \partial x_{j}$.

Рассмотрим $I_{1}$. Согласно теореме 1 ,

$$
\mathbf{E}\left(I_{1}\right)^{2} \leqslant \frac{C}{n \tilde{\delta}}+C \tilde{\delta} .
$$

Запишем следующее очевидное представление для $I_{2}$ :

$$
\begin{aligned}
I_{2}=\{ & \sum_{l=1}^{M} \sum_{k=1}^{N}\left[u_{n}\left(\bar{B}_{n}\left((l-1) \tilde{\delta}+(k-1) h_{n}, \omega\right)-u_{n}\left(\bar{B}_{n}((l-1) \tilde{\delta}, \omega)\right)\right]\right. \\
& \times\left[B_{n}^{j}\left((l-1) \tilde{\delta}+k h_{n}, \omega\right)-B_{n}^{j}\left((l-1) \tilde{\delta}+(k-1) h_{n}, \omega\right)\right] \\
& \left.-\frac{1}{2} \sum_{l=1}^{M} u_{j n}\left(\bar{B}_{n}((l-1) \tilde{\delta}, \omega)\right)\left[B_{n}^{j}(l \tilde{\delta}, \omega)-B_{n}^{j}((l-1) \tilde{\delta}, \omega)\right]^{2}\right\} \\
+ & \frac{1}{2}\left\{\sum_{l=1}^{M} u_{j n}\left(\bar{B}_{n}((l-1) \tilde{\delta}, \omega)\right)\left[B_{n}^{j}(l \tilde{\delta}, \omega)-B_{n}^{j}((l-1) \tilde{\delta}, \omega)\right]^{2}\right. \\
& \left.\quad-\int_{0}^{t} u_{j}(\bar{B}(s, \omega)) d s\right\}=I_{21}+\frac{1}{2} I_{22}, \quad u_{j n}=\frac{\partial}{\partial x_{j}} u_{n},
\end{aligned}
$$

и рассмотрим каждое слагаемое в отдельности.

Несложно убедиться, что

$$
\begin{aligned}
I_{22}= & \left\{\sum _ { l = 1 } ^ { M } u _ { j n } \left(\bar{B}_{n}((l-1) \tilde{\delta}, \omega)\left[B_{n}^{j}(l \tilde{\delta}, \omega)-B_{n}^{j}((l-1) \tilde{\delta}, \omega)\right]^{2}\right.\right. \\
& \left.-\sum_{l=1}^{M} u_{j}\left(\bar{B}_{n}((l-1) \tilde{\delta}, \omega)\right)\left[B_{n}^{j}(l \tilde{\delta}, \omega)-B_{n}^{j}((l-1) \tilde{\delta}, \omega)\right]^{2}\right\} \\
& +\left\{\sum_{l=1}^{M} u_{j}\left(\bar{B}_{n}((l-1) \tilde{\delta}, \omega)\right)\left[B_{n}^{j}(l \tilde{\delta}, \omega)-B_{n}^{j}((l-1) \tilde{\delta}, \omega)\right]^{2}\right. \\
& \left.-\sum_{l=1}^{M} u_{j}\left(\bar{B}_{n}((l-1) \tilde{\delta}, \omega)\right)\left[B^{j}(l \tilde{\delta}, \omega)-B^{j}((l-1) \tilde{\delta}, \omega)\right]^{2}\right\} \\
& +\left\{\sum_{l=1}^{M} u_{j}\left(\bar{B}_{n}((l-1) \tilde{\delta}, \omega)\right)\left[B^{j}(l \tilde{\delta}, \omega)-B^{j}((l-1) \tilde{\delta}, \omega)\right]^{2}\right.
\end{aligned}
$$




$$
\begin{aligned}
& \left.-\sum_{l=1}^{M} u_{j}(\bar{B}((l-1) \tilde{\delta}, \omega))\left[B^{j}(l \tilde{\delta}, \omega)-B^{j}((l-1) \tilde{\delta}, \omega)\right]^{2}\right\} \\
+ & \left\{\sum_{l=1}^{M} u_{j}(\bar{B}((l-1) \tilde{\delta}, \omega))\left[B^{j}(l \tilde{\delta}, \omega)-B^{j}((l-1) \tilde{\delta}, \omega)\right]^{2}\right. \\
& \left.-\int_{0}^{t} u_{j}(\bar{B}(s, \omega)) d s\right\}=I_{22}^{0}+I_{22}^{1}+I_{22}^{2}+I_{22}^{3} .
\end{aligned}
$$

Рассмотрим $I_{22}^{1}$. Действуем точно так, как и при доказательстве леммы 3. В результате получим оценку

$$
\mathbf{E}\left(I_{22}^{1}\right)^{2} \leqslant \frac{C}{n \tilde{\delta}} .
$$

Для оценки $\mathbf{E}\left(I_{22}^{2}\right)^{2}$ воспользуемся $r$-мерной формулой Лагранжа:

$$
\begin{gathered}
\mathbf{E}\left(I_{22}^{2}\right)^{2} \leqslant C M \sum_{i=1}^{r} \sum_{l=1}^{M} \mathbf{E}\left(\left[B_{n}^{i}((l-1) \tilde{\delta}, \omega)-B^{i}((l-1) \tilde{\delta}, \omega)\right]\right. \\
\left.\times\left[B^{j}(l \tilde{\delta}, \omega)-B^{j}((l-1) \tilde{\delta}, \omega)\right]^{2}\right)^{2} \\
\leqslant C M \sum_{i=1}^{r} \sum_{l=1}^{M}\left\{\mathbf{E}\left[B_{n}^{i}((l-1) \tilde{\delta}, \omega)-B^{i}((l-1) \tilde{\delta}, \omega)\right]^{4}\right. \\
\left.\quad \times \mathbf{E}\left[B^{j}(l \tilde{\delta}, \omega)-B^{j}((l-1) \tilde{\delta}, \omega)\right]^{8}\right\}^{1 / 2} \\
\leqslant \frac{C M^{2} \tilde{\delta}^{2}}{n} \leqslant \frac{C}{n} .
\end{gathered}
$$

Стандартными методами, используя определение интеграла от случайного процесса, несложно получить, что

$$
\mathbf{E}\left(I_{22}^{3}\right)^{2} \leqslant C \Delta
$$

где $\Delta=O(\tilde{\delta})$ при $n \rightarrow \infty, h_{n} \rightarrow 0$.

Используя представление $u_{j n}$ и неравенство Гёльдера, получаем $\mathbf{E}\left(I_{22}^{0}\right)^{2} \leqslant C / n^{2}$.

Итоговая оценка для $I_{22}$ будет следующей:

$$
\mathbf{E}\left(I_{22}\right)^{2} \leqslant \frac{C}{n \tilde{\delta}}+\frac{C}{n}+C \tilde{\delta}+\frac{C}{n^{2}} .
$$

Исследуем $I_{21}$. Для этого воспользуемся формулой Тейлора для функции $u$ :

$$
I_{21}=\sum_{l=1}^{M} \sum_{k=1}^{N} \sum_{\substack{i=1 \\ i \neq j}}^{r} u_{i n}\left(\bar{B}_{n}((l-1) \tilde{\delta}, \omega)\right)
$$




$$
\begin{aligned}
& \times\left[B_{n}^{i}\left((l-1) \tilde{\delta}+(k-1) h_{n}, \omega\right)-B_{n}^{i}((l-1) \tilde{\delta}, \omega)\right] \\
& \times\left[B_{n}^{j}\left((l-1) \tilde{\delta}+k h_{n}, \omega\right)-B_{n}^{j}\left((l-1) \tilde{\delta}+(k-1) h_{n}, \omega\right)\right] \\
& +\sum_{l=1}^{M} \sum_{k=1}^{N} u_{j n}\left(\bar{B}_{n}((l-1) \tilde{\delta}, \omega)\right) \\
& \times\left[B_{n}^{j}\left((l-1) \tilde{\delta}+(k-1) h_{n}, \omega\right)-B_{n}^{i}((l-1) \tilde{\delta}, \omega)\right] \\
& \times\left[B_{n}^{j}\left((l-1) \tilde{\delta}+k h_{n}, \omega\right)-B_{n}^{j}\left((l-1) \tilde{\delta}+(k-1) h_{n}, \omega\right)\right] \\
& +\frac{1}{2} \sum_{l=1}^{M} \sum_{k=1}^{N} \sum_{i=1}^{r} u_{i i n}(\theta)\left[B_{n}^{i}\left((l-1) \tilde{\delta}+(k-1) h_{n}, \omega\right)\right. \\
& \left.-B_{n}^{i}((l-1) \tilde{\delta}, \omega)\right]^{2} \\
& \times\left[B_{n}^{j}\left((l-1) \tilde{\delta}+k h_{n}, \omega\right)-B_{n}^{j}\left((l-1) \tilde{\delta}+(k-1) h_{n}, \omega\right)\right] \\
& +\sum_{l=1}^{M} \sum_{k=1}^{N} \sum_{i, p=1}^{r} u_{i p n}(\theta)\left[B_{n}^{i}\left((l-1) \tilde{\delta}+(k-1) h_{n}, \omega\right)\right. \\
& \left.-B_{n}^{i}((l-1) \tilde{\delta}, \omega)\right] \\
& \times\left[B_{n}^{p}\left((l-1) \tilde{\delta}+(k-1) h_{n}, \omega\right)-B_{n}^{p}((l-1) \tilde{\delta}, \omega)\right] \\
& \times\left[B_{n}^{j}\left((l-1) \tilde{\delta}+k h_{n}, \omega\right)-B_{n}^{j}\left((l-1) \tilde{\delta}+(k-1) h_{n}, \omega\right)\right] \\
& -\frac{1}{2} \sum_{l=1}^{M} u_{j n}\left(\bar{B}_{n}((l-1) \tilde{\delta}, \omega)\right)\left[B_{n}^{j}(l \tilde{\delta}, \omega)-B_{n}^{j}((l-1) \tilde{\delta}, \omega)\right]^{2} \\
& =I_{21}^{1}+I_{21}^{2}+I_{21}^{3}+I_{21}^{4}, \quad u_{i p n}=\frac{\partial^{2}}{\partial x_{i} \partial x_{p}} u_{n} .
\end{aligned}
$$

Рассмотрим $I_{21}^{1}$. Положим по определению

$$
\begin{aligned}
\bar{B}_{n}^{\prime}\left((l-1) \tilde{\delta}-\frac{1}{n}, \omega\right)= & \left(B_{n}^{1}((l-1) \tilde{\delta}, \omega), B_{n}^{2}((l-1) \tilde{\delta}, \omega), \ldots,\right. \\
& \left.B_{n}^{j}\left((l-1) \tilde{\delta}-\frac{1}{n}, \omega\right), \ldots, B_{n}^{r}((l-1) \tilde{\delta}, \omega)\right) .
\end{aligned}
$$

Несложно видеть, что в этом случае $u_{i n}\left(B_{n}^{\prime}((l-1) \tilde{\delta}-1 / n, \omega)\right)$ независит от третьего сомножителя в $I_{21}^{1}$. Очевидно следующее представление:

$$
\begin{aligned}
I_{21}^{1}= & I_{21}^{1}-\sum_{l=1}^{M} \sum_{k=1}^{N} \sum_{\substack{i=1 \\
i \neq j}}^{r} u_{i n}\left(B_{n}^{\prime}\left((l-1) \tilde{\delta}-\frac{1}{n}, \omega\right)\right) \\
& \times\left[B_{n}^{i}\left((l-1) \tilde{\delta}+(k-1) h_{n}, \omega\right)-B_{n}^{i}((l-1) \tilde{\delta}, \omega)\right]
\end{aligned}
$$




$$
\begin{aligned}
& \times\left[B_{n}^{j}\left((l-1) \tilde{\delta}+k h_{n}, \omega\right)-B_{n}^{j}\left((l-1) \tilde{\delta}+(k-1) h_{n}, \omega\right)\right] \\
& +\sum_{l=1}^{M} \sum_{k=1}^{N} \sum_{\substack{i=1 \\
i \neq j}}^{r} u_{i n}\left(B_{n}^{\prime}\left((l-1) \tilde{\delta}-\frac{1}{n}, \omega\right)\right) \\
& \times\left[B_{n}^{i}\left((l-1) \tilde{\delta}+(k-1) h_{n}, \omega\right)-B_{n}^{i}((l-1) \tilde{\delta}, \omega)\right] \\
& \times\left[B_{n}^{j}\left((l-1) \tilde{\delta}+k h_{n}, \omega\right)-B_{n}^{j}\left((l-1) \tilde{\delta}+(k-1) h_{n}, \omega\right)\right] \\
& =I_{21}^{11}+I_{21}^{12} .
\end{aligned}
$$

Пользуясь независимостью сомножителей, получаем

$$
\begin{aligned}
\mathbf{E}\left(I_{21}^{12}\right)^{2} \leqslant & C M N \mathbf{E}\left[B_{n}^{i}\left((l-1) \tilde{\delta}+(k-1) h_{n}, \omega\right)-B_{n}^{i}((l-1) \tilde{\delta}, \omega)\right]^{2} \\
& \times \mathbf{E}\left[B_{n}^{j}\left((l-1) \tilde{\delta}+k h_{n}, \omega\right)-B_{n}^{j}\left((l-1) \tilde{\delta}+(k-1) h_{n}, \omega\right)\right]^{2} \\
\leqslant & C \tilde{\delta} n h_{n} .
\end{aligned}
$$

Используя формулу Лагранжа и ограниченность на $\mathbf{R}^{r}$ вторых частных производных $u$, имеем

$$
\begin{aligned}
\mathbf{E}\left(I_{21}^{11}\right)^{2} \leqslant & C M^{2} N^{2} \mathbf{E}\left\{\left[B_{n}^{j}((l-1) \tilde{\delta}, \omega)-B_{n}^{j}\left((l-1) \tilde{\delta}-\frac{1}{n}, \omega\right)\right]^{2}\right. \\
& \left.\times\left[B_{n}^{j}\left((l-1) \tilde{\delta}+k h_{n}, \omega\right)-B_{n}^{j}\left((l-1) \tilde{\delta}+(k-1) h_{n}, \omega\right)\right]^{2}\right\} \\
& \times \mathbf{E}\left[B_{n}^{i}\left((l-1) \tilde{\delta}+(k-1) h_{n}, \omega\right)-B_{n}^{i}((l-1) \tilde{\delta}, \omega)\right]^{2} \\
\leqslant & C \tilde{\delta}+C n^{3} h_{n}^{3} \tilde{\delta} .
\end{aligned}
$$

Таким образом, $\mathbf{E}\left(I_{21}^{1}\right)^{2} \leqslant C \tilde{\delta}$.

Легко установить справедливость следующего тождества:

$$
\begin{aligned}
\sum_{k=1}^{N} & {\left[B_{n}^{j}\left((l-1) \tilde{\delta}+(k-1) h_{n}, \omega\right)-B_{n}^{j}((l-1) \tilde{\delta}, \omega)\right] } \\
& \times\left[B_{n}^{j}\left((l-1) \tilde{\delta}+k h_{n}, \omega\right)-B_{n}^{j}\left((l-1) \tilde{\delta}+(k-1) h_{n}, \omega\right)\right] \\
= & \frac{1}{2}\left[B_{n}^{j}(l \tilde{\delta}, \omega)-B_{n}^{j}((l-1) \tilde{\delta}, \omega)\right]^{2} \\
& -\frac{1}{2} \sum_{k=1}^{N}\left[B_{n}^{j}\left((l-1) \tilde{\delta}+k h_{n}, \omega\right)-B_{n}^{j}\left((l-1) \tilde{\delta}+(k-1) h_{n}, \omega\right)\right]^{2}
\end{aligned}
$$


Используя это тождество и лемму 2 , получаем

$$
\begin{aligned}
\mathbf{E}\left(I_{21}^{2}\right)^{2} \leqslant & \frac{1}{4} \mathbf{E}\left\{\sum_{l=1}^{M} \sum_{k=1}^{N} u_{j}\left(\bar{B}_{n}((l-1) \tilde{\delta}, \omega)\right)\right. \\
& \left.\times\left[B_{n}^{j}\left((l-1) \tilde{\delta}+k h_{n}, \omega\right)-B_{n}^{j}\left((l-1) \tilde{\delta}+(k-1) h_{n}, \omega\right)\right]^{2}\right\}^{2} \\
\leqslant & C M^{2} N^{2} n^{2} h_{n}^{4} \leqslant C n^{2} h_{n}^{2} .
\end{aligned}
$$

Учитывая ограниченность функции $u$ и ее частных производных, для $\mathrm{E}\left(I_{21}^{3}\right)^{2}$ и $\mathrm{E}\left(I_{21}^{4}\right)^{2}$ получаем

$$
\mathrm{E}\left(I_{21}^{z}\right)^{2} \leqslant C n \tilde{\delta}^{2}, \quad z=3,4 .
$$

Тогда для $\mathbf{E}\left(I_{21}\right)^{2}$ получаем ощенку

$$
\mathbf{E}\left(I_{21}\right)^{2} \leqslant C \tilde{\delta} n h_{n}+C \tilde{\delta}+C n \tilde{\delta}^{2}+C n^{2} h_{n}^{2} .
$$

В итоге получена следуюшая оценка:

$$
\begin{gathered}
\mathbf{E}\left\{\sum_{k=1}^{p} u_{n}\left(\bar{B}_{n}\left(t_{k-1}, \omega\right)\right)\left[B_{n}^{j}\left(t_{k}, \omega\right)-B_{n}^{j}\left(t_{k-1}, \omega\right)\right]\right. \\
\left.\quad-(\mathrm{S}) \int_{0}^{t} u(\bar{B}(s, \omega)) d B^{j}(s, \omega)\right\}^{2} \\
\leqslant \frac{C}{n \tilde{\delta}}+C \tilde{\delta}+C \tilde{\delta} n h_{n}+C n \tilde{\delta}^{2}+C n^{2} h_{n}^{2} .
\end{gathered}
$$

В завершение доказательства теоремы укажем, что оптимальный результат ощенивания достигается для $\tilde{\delta}=n^{-2 / 3}$.

Следствие. ІІусть выполнены условия теоремы 3 , тогда если $n \rightarrow \infty, h_{n} \rightarrow 0$ max, umo $h_{n}=o(1 / n)$, mo

$$
\begin{gathered}
\sup _{t \in T} \mathrm{E}\left\{\sum_{i=1}^{r} \sum_{k=1}^{p} u_{n}\left(\bar{B}_{n}\left(t_{k-1}, \omega\right)\right)\left[B_{n}^{i}\left(t_{k}, \omega\right)-B_{n}^{i}\left(t_{k-1}, \omega\right)\right]\right. \\
\left.-\sum_{i=1}^{r}(\mathrm{~S}) \int_{0}^{t} u(\bar{B}(s, \omega)) d B^{i}(s, \omega)\right\}^{2} \longrightarrow 0 .
\end{gathered}
$$

Для случая интеграла Стратоновича справедливо утверждение, аналогичное теореме 2.

Теорема 4. Пусть функция $u \in C^{1}\left(\mathbf{R}^{r}\right)$ u $\bar{B}(t, \omega)$ есть $r$-мерлый стандартный случайный проиесс $\mathcal{F}_{t}$-броуновского движения. Тогда найдутся такие элементы $\widetilde{u} \in \mathcal{G}\left(\mathbf{R}^{r}\right)$ u $\widetilde{B} \in \mathcal{G}(\widetilde{T}, \Omega)^{r}$, что если для любого сколь угодно малого $\alpha>0$ при $n \rightarrow \infty, h_{n} \rightarrow 0$

$$
h_{n}=o\left(\frac{1}{n^{1+\alpha}}\right)
$$


mo

$$
\begin{aligned}
\sup _{t \in T} \mid & \sum_{i=1}^{r} \sum_{k=1}^{p} u_{n}\left(\bar{B}_{n}\left(t_{k-1}, \omega\right)\right)\left[B_{n}^{i}\left(t_{k}, \omega\right)-B_{n}^{i}\left(t_{k-1}, \omega\right)\right] \\
& -\sum_{i=1}^{r}(S) \int_{0}^{t} u(\bar{B}(s, \omega)) d B^{i}(s, \omega) \mid \longrightarrow 0
\end{aligned}
$$

для почти всех $\omega \in \Omega$. Здесь $\left(u_{n}\right)$ u $\left(\bar{B}_{n}\right)$ - произвольнье представители и и $\widetilde{B}$ соотөетственно.

4. Смешанный случай. В этом пункте мы рассматриваем задачу аппроксимации одновременно стохастического интеграла Ито и стохастического интеграла Стратоновича конечными суммами элементов из прямого произведения алгебр обобщенных случайных процессов. ІІодобные исследования, насколько нам известно, проводятся впервые.

Рассмотрим случай $r=2$. Пусть обобщенный случайный процесс броуновского движения $\widetilde{B}(t, \omega)$ из $\mathcal{G}(T, \Omega)^{2}$ имеет вид: $\widetilde{B}=\left[\left(\bar{B}_{n}\right)\right]=$ $\left(\left[\left(B_{n}^{1}\right)\right],\left[\left(B_{n}^{2}\right)\right]\right)$, где $B_{n}^{1}(t, \omega)=\left(B^{1} * \rho_{n}\right)(t, \omega), B_{n}^{2}(t, \omega)=\left(B^{2} * \rho_{n}^{1}\right)(t, \omega)$, $\rho_{n}(\dot{t})$ из $(1.1)$, а $\rho_{n}^{1}(t)=\varphi(n) \rho(\varphi(n) t), n \in \mathbf{N}, \varphi: \mathbf{R} \rightarrow \mathbf{R}$ - монотонно неубывающая функция. Тогда справедливы следующие утверждения.

Теорема 5. Для любой $u \in C_{B}^{2}\left(\mathbf{R}^{2}\right)$ и двумерного стандартного случайного прочесса $\mathcal{F}_{t}$-броуновского движения $\bar{B}(t, \omega)$ справедливо неравенство

$$
\begin{aligned}
\sup _{t \in T} \mathbf{E} & \left\{\sum_{i=1}^{2} \sum_{k=1}^{p} u_{n}\left(\bar{B}_{n}\left(t_{k-1}, \omega\right)\right)\left[B_{n}^{i}\left(t_{k}, \omega\right)-B_{n}^{i}\left(t_{k-1}, \omega\right)\right]\right. \\
& \left.\quad-(\mathrm{S}) \int_{0}^{t} u(\bar{B}(s, \omega)) d B^{1}(s, \omega)-(\mathrm{I}) \int_{0}^{t} u(\bar{B}(s, \omega)) d B^{2}(s, \omega)\right\}^{2} \\
\leqslant & \frac{C}{n^{1 / 3}}+C h_{n}+C n^{2} h_{n}^{2}+\frac{C}{\varphi(n) h_{n}}
\end{aligned}
$$

где $u_{n}(s)=\left(u * \bar{\rho}_{n}\right)(s), a \bar{\rho}_{n} u 3(2.3)$.

Доказ а тельство. Рассмотрим сначала случай Ито. Используя ту же методику доказательства, что и в теореме 1 , получаем, чTо

$$
\begin{gathered}
\mathrm{E}\left\{\sum_{k=1}^{p} u_{n}\left(\bar{B}_{n}\left(t_{k-1}, \omega\right)\right)\left[B_{n}^{2}\left(t_{k}, \omega\right)-B_{n}^{2}\left(t_{k-1}, \omega\right)\right]\right. \\
\left.\quad-(\mathrm{I}) \int_{0}^{t} u(\bar{B}(s, \omega)) d B^{2}(s, \omega)\right\}^{2} \\
\leqslant \frac{C}{\varphi(n) h_{n}}+\frac{C}{\varphi(n)}+C h_{n}+\frac{C}{n^{2} h_{n}}
\end{gathered}
$$


А теперь оценим разность

$$
\begin{aligned}
& \sum_{k=1}^{p} u_{n}\left(\bar{B}_{n}\left(t_{k-1}, \omega\right)\right)\left[B_{n}^{1}\left(t_{k}, \omega\right)-B_{n}^{1}\left(t_{k-1}, \omega\right)\right] \\
& -(\mathrm{S}) \int_{0}^{t} u(\bar{B}(s, \omega)) d B^{1}(s, \omega)
\end{aligned}
$$

Выберем такое $N=N\left(h_{n}\right):(0,1) \longrightarrow \mathbf{Z}^{+}$, что $h_{n} N\left(h_{n}\right)=\tilde{\delta} \longrightarrow 0$, $N\left(h_{n}\right) \rightarrow \infty, 1 / n=o(\tilde{\delta})$ при $h_{n} \rightarrow 0$. Без существенного ограничения общности предположим, что $t=p h_{n}=N M h_{n}=M \tilde{\delta}$.

Несложно видеть, что

$$
\begin{aligned}
\sum_{k=1}^{p} u_{n}\left(\bar{B}_{n}\left(t_{k-1}, \omega\right)\right)\left[B_{n}^{1}\left(t_{k}, \omega\right)-B_{n}^{1}\left(t_{k-1}, \omega\right)\right] & -(\mathrm{S}) \int_{0}^{t} u(\bar{B}(s, \omega)) d B^{1}(s, \omega) \\
= & \left\{\sum_{l=1}^{M} u_{n}\left(\bar{B}_{n}((l-1) \tilde{\delta}, \omega)\right)\left[B_{n}^{1}(l \tilde{\delta}, \omega)-B_{n}^{1}((l-1) \tilde{\delta}, \omega)\right]\right. \\
& \left.-(\mathrm{I}) \int_{0}^{t} u(\bar{B}(s, \omega)) d B^{1}(s, \omega)\right\} \\
+ & \left\{\sum _ { l = 1 } ^ { M } \sum _ { k = 1 } ^ { N } \left[u_{n}\left(\bar{B}_{n}\left((l-1) \tilde{\delta}+(k-1) h_{n}, \omega\right)\right)\right.\right. \\
& \times\left[B_{n}^{1}\left((l-1) \tilde{\delta}+k h_{n}, \omega\right)-B_{n}^{1}\left((l-1) \tilde{\delta}+(k-1) h_{n}, \omega\right)\right] \\
& \left.-\frac{1}{2} \int_{0}^{t} \frac{\partial u}{\partial x_{1}}\left(\bar{B}_{n}(s, \omega)\right) d s\right\}=J_{1}+J_{2} .
\end{aligned}
$$

Для $J_{1}$, используя (4.1), получаем следующую оценку:

$$
\mathbf{E}\left[J_{1}\right]^{2} \leqslant \frac{C}{n^{2} \tilde{\delta}}+\frac{C}{n \tilde{\delta}}+C \tilde{\delta}+\frac{C}{n} .
$$

Iредставим слагаемое $J_{2}$ в виде

$$
\begin{aligned}
J_{2}=\{ & \sum_{l=1}^{M} \sum_{k=1}^{N}\left[u_{n}\left(\bar{B}_{n}\left((l-1) \tilde{\delta}+(k-1) h_{n}, \omega\right)\right)-u_{n}\left(\bar{B}_{n}((l-1) \tilde{\delta}, \omega)\right)\right] \\
& \times\left[B_{n}^{1}\left((l-1) \tilde{\delta}+k h_{n}, \omega\right)-B_{n}^{1}\left((l-1) \tilde{\delta}+(k-1) h_{n}, \omega\right)\right] \\
& \left.-\frac{1}{2} \sum_{l=1}^{M} \frac{\partial u_{n}}{\partial x_{1}}\left(B_{n}((l-1) \tilde{\delta}, \omega)\right)\left[B_{n}^{1}(l \tilde{\delta}, \omega)-B_{n}^{1}((l-1) \tilde{\delta}, \omega)\right]^{2}\right\}
\end{aligned}
$$




$$
\begin{gathered}
+\frac{1}{2}\left\{\sum_{l=1}^{M} \frac{\partial u_{n}}{\partial x_{1}}\left(\bar{B}_{n}((l-1) \tilde{\delta}, \omega)\right)\left[B_{n}^{1}(l \tilde{\delta}, \omega)-B_{n}^{1}((l-1) \tilde{\delta}, \omega)\right]^{2}\right. \\
\left.\quad-\int_{0}^{t} \frac{\partial u}{\partial x_{1}}(\bar{B}(s, \omega)) d s\right\}=I_{21}+\frac{1}{2} I_{22}
\end{gathered}
$$

Применяя методику, предложенную в доказательстве теоремы 3, получаем следующую оценку для $\mathrm{E}\left[I_{22}\right]^{2}$ :

$$
\mathrm{E}\left[I_{22}\right]^{2} \leqslant C\left(\frac{1}{n}+\frac{1}{\varphi(n)}\right)+\frac{C}{n \tilde{\delta}}+C \Delta,
$$

где $\Delta=O(\tilde{\delta})$.

Исследуем теперь $I_{21}$. Применяя многомерную формулу Тейлора, можем получить

$$
\begin{aligned}
I_{21} \leqslant & \left\{\sum _ { l = 1 } ^ { M } \sum _ { k = 1 } ^ { N } \left\{\frac{\partial u_{n}}{\partial x_{1}}\left(\bar{B}_{n}((l-1) \tilde{\delta}, \omega)\right)\right.\right. \\
& \times\left[B_{n}^{1}\left((l-1) \tilde{\delta}+(k-1) h_{n}, \omega\right)-B_{n}^{1}((l-1) \tilde{\delta}, \omega)\right] \\
& \left.\times\left[B_{n}^{1}\left((l-1) \tilde{\delta}+k h_{n}, \omega\right)-B_{n}^{1}\left((l-1) \tilde{\delta}+(k-1) h_{n}, \omega\right)\right]\right\} \\
& \left.-\frac{1}{2} \sum_{l=1}^{M} \frac{\partial u_{n}}{\partial x_{1}}\left(\bar{B}_{n}((l-1) \tilde{\delta}, \omega)\right)\left[B_{n}^{1}(l \tilde{\delta}, \omega)-B_{n}^{1}((l-1) \tilde{\delta}, \omega)\right]^{2}\right\} \\
+ & \left\{\sum_{l=1}^{M} \sum_{k=1}^{N} \frac{\partial u_{n}}{\partial x_{2}}\left(\bar{B}_{n}((l-1) \tilde{\delta}, \omega)\right)\right. \\
& \times\left[B_{n}^{2}\left((l-1) \tilde{\delta}+(k-1) h_{n}, \omega\right)-B_{n}^{2}((l-1) \tilde{\delta}, \omega)\right] \\
& \times\left[B_{n}^{1}\left((l-1) \tilde{\delta}+k h_{n}, \omega\right)-B_{n}^{1}\left((l-1) \tilde{\delta}+(k-1) h_{n}, \omega\right)\right] \\
+ & +2 \sum_{l=1}^{1} \sum_{k=1}^{N} \frac{\partial^{2} u_{n}}{\partial x_{1}^{2}}\left(\theta_{1}^{2}\right)\left[B_{n}^{1}\left((l-1) \tilde{\delta}+(k-1) h_{n}^{3}, \omega\right)-B_{n}^{1}((l-1) \tilde{\delta}, \omega)\right]^{2} \\
\times & {\left[B_{n}^{1}\left((l-1) \tilde{\delta}+k h_{n}, \omega\right)-B_{n}^{1}\left((l-1) \tilde{\delta}+(k-1) h_{n}, \omega\right)\right] } \\
+ & \\
+ & \\
+ &
\end{aligned}
$$


Применяя неравенство Гёльдера, для $I_{21}^{3}$ находим, что

$$
\begin{aligned}
\mathbf{E}\left(I_{21}^{3}\right)^{2} \leqslant & M N \sum_{l=1}^{M} \sum_{k=1}^{N} \mathbf{E}\left\{\left[B_{n}^{1}\left((l-1) \tilde{\delta}+(k-1) h_{n}, \omega\right)\right.\right. \\
& \left.-B_{n}^{1}((l-1) \tilde{\delta}, \omega)\right]^{2} \\
\times & {\left.\left[B_{n}^{1}\left((l-1) \tilde{\delta}+k h_{n}, \omega\right)-B_{n}^{1}\left((l-1) \tilde{\delta}+(k-1) h_{n}, \omega\right)\right]\right\}^{2} } \\
\leqslant & C M^{2} N^{2} \tilde{\delta}^{2} n h_{n}^{2} \leqslant C n \tilde{\delta}^{2} .
\end{aligned}
$$

Аналогичная оценка получается для $\mathbf{E}\left(I_{21}^{4}\right)^{2}$.

Представим $I_{21}^{2}$ в виде:

$$
\begin{aligned}
I_{21}^{2}= & I_{21}^{2}-\sum_{l=1}^{M} \sum_{k=1}^{N} \frac{\partial u_{n}}{\partial x_{2}}\left(B_{n}^{1}\left((l-1) \tilde{\delta}-\frac{1}{n}, \omega\right), B_{n}^{2}\left((l-1) \tilde{\delta}-\frac{1}{\varphi(n)}, \omega\right)\right) \\
& \times\left[B_{n}^{2}\left((l-1) \tilde{\delta}+(k-1) h_{n}, \omega\right)-B_{n}^{2}((l-1) \tilde{\delta}, \omega)\right] \\
& \times\left[B_{n}^{1}\left((l-1) \tilde{\delta}+k h_{n}, \omega\right)-B_{n}^{1}\left((l-1) \tilde{\delta}+(k-1) h_{n}, \omega\right)\right] \\
& +\sum_{l=1}^{M} \sum_{k=1}^{N} \frac{\partial u_{n}}{\partial x_{2}}\left(B_{n}^{1}\left((l-1) \tilde{\delta}-\frac{1}{n}, \omega\right), B_{n}^{2}\left((l-1) \tilde{\delta}-\frac{1}{\varphi(n)}, \omega\right)\right) \\
& \times\left[B_{n}^{2}\left((l-1) \tilde{\delta}+(k-1) h_{n}, \omega\right)-B_{n}^{2}((l-1) \tilde{\delta}, \omega)\right] \\
& \times\left[B_{n}^{1}\left((l-1) \tilde{\delta}+k h_{n}, \omega\right)-B_{n}^{1}\left((l-1) \tilde{\delta}+(k-1) h_{n}, \omega\right)\right]=I_{21}^{21}+I_{21}^{22} .
\end{aligned}
$$

Оценим $I_{21}^{21}$ и $I_{21}^{22}$ :

$$
\begin{aligned}
\mathbf{E}\left(I_{21}^{21}\right)^{2} & \leqslant C M N \sum_{l=1}^{M} \sum_{k=1}^{N} \mathbf{E}\left\{\left[B_{n}^{1}((l-1) \tilde{\delta}, \omega)-B_{n}^{1}\left((l-1) \tilde{\delta}-\frac{1}{n}, \omega\right)\right]\right. \\
\times & {\left[B_{n}^{2}\left((l-1) \tilde{\delta}+(k-1) h_{n}, \omega\right)-B_{n}^{2}((l-1) \tilde{\delta}, \omega)\right] } \\
\times & {\left.\left[B_{n}^{1}\left((l-1) \tilde{\delta}+k h_{n}, \omega\right)-B_{n}^{1}\left((l-1) \tilde{\delta}+(k-1) h_{n}, \omega\right)\right]\right\}^{2} } \\
+ & C M N \sum_{l=1}^{M} \sum_{k=1}^{N} \mathbf{E}\left\{\left[B_{n}^{2}((l-1) \tilde{\delta}, \omega)-B_{n}^{2}\left((l-1) \tilde{\delta}-\frac{1}{\varphi(n)}, \omega\right)\right]\right. \\
\times & {\left[B_{n}^{2}\left((l-1) \tilde{\delta}+(k-1) h_{n}, \omega\right)-B_{n}^{2}((l-1) \tilde{\delta}, \omega)\right] } \\
\times & {\left.\left[B_{n}^{1}\left((l-1) \tilde{\delta}+k h_{n}, \omega\right)-B_{n}^{1}\left((l-1) \tilde{\delta}+(k-1) h_{n}, \omega\right)\right]\right\}^{2} } \\
\leqslant & C \tilde{\delta}+\frac{C \tilde{\delta} n}{\varphi(n)} \leqslant C \tilde{\delta}
\end{aligned}
$$




$$
\begin{aligned}
\mathbf{E}\left(I_{21}^{22}\right)^{2} \leqslant & C \sum_{l=1}^{M} \sum_{k=1}^{N} \mathbf{E}\left[B_{n}^{2}\left((l-1) \tilde{\delta}+(k-1) h_{n}, \omega\right)-B_{n}^{2}((l-1) \tilde{\delta}, \omega)\right]^{2} \\
& \times \mathbf{E}\left[B_{n}^{1}\left((l-1) \tilde{\delta}+k h_{n}, \omega\right)-B_{n}^{1}\left((l-1) \tilde{\delta}+(k-1) h_{n}, \omega\right)\right]^{2} \\
\leqslant & C M N \tilde{\delta} n h_{n}^{2} \leqslant C n \tilde{\delta} h_{n} .
\end{aligned}
$$

Рассмотрим $I_{21}^{1}$. Воспользуемся следующим тождеством:

$$
\begin{aligned}
\sum_{k=1}^{N} & {\left[B_{n}^{1}\left((l-1) \tilde{\delta}+(k-1) h_{n}, \omega\right)-B_{n}^{1}((l-1) \tilde{\delta}, \omega)\right] } \\
& \times\left[B_{n}^{1}\left((l-1) \tilde{\delta}+k h_{n}, \omega\right)-B_{n}^{1}\left((l-1) \tilde{\delta}+(k-1) h_{n}, \omega\right)\right] \\
= & \frac{1}{2}\left[B_{n}^{1}(l \tilde{\delta}, \omega)-B_{n}^{1}((l-1) \tilde{\delta}, \omega)\right]^{2} \\
& -\frac{1}{2} \sum_{k=1}^{N}\left[B_{n}^{1}\left((l-1) \tilde{\delta}+k h_{n}, \omega\right)-B_{n}^{1}\left((l-1) \tilde{\delta}+(k-1) h_{n}, \omega\right)\right]^{2} .
\end{aligned}
$$

В результате имеем:

$$
\begin{gathered}
\mathbf{E}\left(I_{21}^{1}\right)^{2} \leqslant C M N \sum_{l=1}^{M} \sum_{k=1}^{N} \mathbf{E}\left[B_{n}^{1}\left((l-1) \tilde{\delta}+k h_{n}, \omega\right)\right. \\
\left.\quad-B_{n}^{1}\left((l-1) \tilde{\delta}+(k-1) h_{n}, \omega\right)\right]^{4} \\
\leqslant C M^{2} N^{2} n^{2} h_{n}^{4} \leqslant C n^{2} h_{n}^{2} .
\end{gathered}
$$

Таким образом,

$$
\mathbf{E}\left(I_{21}\right)^{2} \leqslant C n \tilde{\delta}+C \tilde{\delta}+C n^{2} h_{n}^{2}
$$

Следствие. Пусть выполнены условия теоремы 5. Тогда, если $\varphi(n) \rightarrow \infty, h_{n} \rightarrow 0$ npu $n \rightarrow \infty$ max, umo $h_{n}=o(1 / n), 1 / \varphi(n)=o\left(h_{n}\right)$, mo

$$
\begin{array}{rl}
\sup _{t \in T} & \mathbf{E}\left\{\sum_{i=1}^{2} \sum_{k=1}^{p} u_{n}\left(\bar{B}_{n}\left(t_{k-1}, \omega\right)\right)\left[B_{n}^{i}\left(t_{k}, \omega\right)-B_{n}^{i}\left(t_{k-1}, \omega\right)\right]\right. \\
& \left.-(\mathrm{S}) \int_{0}^{t} u(\bar{B}(s, \omega)) d B^{1}(s, \omega)-(\mathrm{I}) \int_{0}^{t} u(\bar{B}(s, \omega)) d B^{2}(s, \omega)\right\}^{2} \longrightarrow 0 .
\end{array}
$$

Теорема 6. Пусть функция $u \in C_{B}^{1}\left(\mathbf{R}^{2}\right)$, a $\bar{B}(t, \omega)-$ двумерный стандартный случайный прочесс $\mathcal{F}_{t}$-броуновского движения. Тогда если для любых сколь угодно мальх $\alpha>0$ и $\delta>0 \varphi(n) \rightarrow \infty, h_{n} \rightarrow 0$ при $n \rightarrow \infty$ max, umo

$$
h_{n}=o\left(\frac{1}{n^{1+\alpha}}\right), \quad \frac{1}{\varphi(n)}=o\left(h_{n}^{1+\delta}\right),
$$


mo

$$
\begin{aligned}
\sup _{t \in T} & \mid \sum_{i=1}^{2} \sum_{k=1}^{p} u_{n}\left(\bar{B}_{n}\left(t_{k-1}, \omega\right)\right)\left[B_{n}^{i}\left(t_{k}, \omega\right)-B_{n}^{i}\left(t_{k-1}, \omega\right)\right] \\
& -(\mathrm{S}) \int_{0}^{t} u(\bar{B}(s, \omega)) d B^{1}(s, \omega)-\left.(\mathrm{I}) \int_{0}^{t} u(\bar{B}(s, \omega)) d B^{2}(s, \omega)\right|^{2} \longrightarrow 0
\end{aligned}
$$

для почти всех $\omega \in \Omega$. Здесь $u_{n} u \bar{B}_{n}=\left(B_{n}^{1}, B_{n}^{2}\right)$ maкuе же, как и meopene 5 .

3 а м е ч а н и е. Пусть функция $u \in C^{2}(\mathbf{R}), B-$ одномерный стандартный процесс $\mathcal{F}_{t}$-броуновского движения,

$$
u_{n}=u * \rho_{n}, \quad B_{n}^{\prime}=B * \rho_{n}, \quad B_{n}^{\prime \prime}=B * \rho_{n}^{1},
$$

а $\rho_{n}$ и $\rho_{n}^{\mathbf{1}}$ выбираем такими же, как и в теореме 5 . Тогда из теорем 1 и 3 вытекает, что выражения

$$
\begin{aligned}
& S_{n p}^{m}(t, \omega)=\sum_{k=1}^{p} u_{n}\left(B_{n}^{\prime}\left(t_{k-1}, \omega\right)\right)\left[B_{n}^{\prime}\left(t_{k}, \omega\right)-B_{n}^{\prime}\left(t_{k-1}, \omega\right)\right] \\
& I_{n p}^{m}(t, \omega)=\sum_{k=1}^{p} u_{n}\left(B_{n}^{\prime \prime}\left(t_{k-1}, \omega\right)\right)\left[B_{n}^{\prime \prime}\left(t_{k}, \omega\right)-B_{n}^{\prime \prime}\left(t_{k-1}, \omega\right)\right]
\end{aligned}
$$

при $n \rightarrow \infty, m \rightarrow \infty, \lambda_{m} \rightarrow 0$ аппроксимируют стохастический интеграл

$$
\int_{0}^{t} u(B(s, \omega)) d B(s, \omega), \quad t \in T
$$

понимаемый, соответственно, в смысле Ито или Стратоновича.

Таким образом, учитывая связь между стохастическими интегралами Ито и Стратоновича (см. доказательство теоремы 3 ), получаем, что при $n \rightarrow \infty, m \rightarrow \infty, \lambda_{m} \rightarrow 0$ разность $S_{n p}^{m}-I_{n p}^{m}$ сходится в $L^{2}(\Omega, \mathcal{A}, \mathbf{P})$ равномерно по $t \in T$ к интегралу

$$
\frac{1}{2} \int_{0}^{t} u^{\prime}(B(s, \omega)) d s
$$

Подобная ситуация возникла в результате того, что почти все траектории случайного процесса $B$ имеют неограниченную вариацию на любом отрезке. Для случайных процессов с более гладкими траекториями поведение разности будет иным. Так, например, если траектории случайного прощесса в интеграле типа $(4.2)$ - из класса $C^{\mathbf{1}}(\mathbf{R})$, то рассматриваемая разность будет сходиться к нулю.

В общем случае предельное поведение выражения вида $S_{n p}^{m}-I_{n p}^{m}$ при $n \rightarrow \infty, m \rightarrow \infty, \lambda_{m} \rightarrow 0$ будет зависеть от степени гладкости функций в стохастическом интеграле и способа их апщроксимащии. 


\section{СПИСОК ЛИТЕРАТУРЫ}

1. Schwartz L. Théorie des distributions. I-II. Paris: Hermann, 1950.

2. Dirac $P$. A. Theory of emission and absorption of radiation. - Proc. Roy. Soc. Serie A, 1927 , v. 114, p. 243-262.

3. Mikusinski J. On the square of the Dirac delta distribution. - Bull. Acad. Polish. Sci., 1966 , v. 14 , № 9, p. 511-513.

4. Антосик П., Микусинский Я., Сикорский $Р$. Теория обобщенных функций: секвенциальный подход. М.: Мир, 1976, 311 с.

5. Ливчак Я. Б. К теории обобщенных функций. - Труды Рижского алгебр. семинара, 1969, с. $98-164$.

6. Colombeau J. F. A multiplication of distributions. - J. Math. Anal. Appl., 1983, v. 94, № 1 , p. $96-115$.

7. Oberguggenberger $M$. Distributions associated with multiplication of distributions in the Colombeau algebra. - Boll. unione mat. ital., 1986, t. 5-A, № 6, p. 423-429.

8. Todorov T. D. Sequential approach to Colombeau's theory of generalized functions. International Centre for Theoretical Physics. Trieste, 1987, v. 126.

9. Еәоров Ю. В. К теории обобщенных функций. - Успехи матем. наук, 1990, т. 45, B. 5, c. 3-40.

10. Антоневич А. Б., Радыно Я. В. Об обтем методе построения алгебр обобценных функций. - Докл. АН СССР, 1991, т. 318, № 2, с. 267-270.

11. Гельфанд И. М. Обобщенные случайные процессы. - Докл. АН СССР, 1955, т. 100 , № 5, с. 853-856.

12. Гельфаяд И. М., Виленкин Н. Я. Некоторые применения гармонического анализа. Оснащенные гильбертовы пространства. (Обобщенные функции. В. 4.) М.: Физматгиз, 1961, 472 с.

13. Урбаник $K$. Случайные процессы, реализации которых являются обобщенными функциями. - Теория вероятн. и ее примен., 1956, т. I, в. 1, с. 146-149.

14. Лазахович $H$. В. Стохастические дифференциалы в алгебре обобщенных случайных процессов. - Докл. АН Беларуси, 1994, т. 38, № 5, с. 23-27.

15. Лазакович $H$. В. Аппроксимахия стохастических дифференциальных уравнений конечно-разностными. - Докл. АН Беларуси, 1995, т. 39, № 3, с. 20-22.

16. Ватанабэ $C$., Икэда $H$. Стохастические дифференциальные уравнения и диффузионные процессы. М.: Наука, 1986, 448 с.

17. Липцер P. III., IIиряев A. Н. Статистика случайных процессов. М.: Наука, 1974, $696 \mathrm{c}$.

18. Веятиель A. Д. Курс теории случайных процессов. М.: Наука, 1975, 320 с.

19. Хида T. Броуновское движение. М.: Наука, 1987, 304 с.

20. Wong $E$., Zakai $M$. On the relationship between ordinary and stochastic differential equations. - Internat. J. Engin. Sci., 1965, v. 3, p. 213-229.

21. Мачкявичюс $B$. Некоторые аппроксимации стохастических интегралов и решений стохастических дифференциальных уравнений. - Литов. матем. сб., 1978, т. 18, № 3, c. 101-108.

22. Розаяов Ю. А. Теория вероятностей, случайные процессы и математическая статистика. М.: Наука, 1989, 320 с. 